\title{
HYBRID SIMULATION CHALLENGES AND OPPORTUNITIES: A LIFE-CYCLE APPROACH
}

\author{
Tillal Eldabi \\ Brunel Business School \\ Brunel University London \\ Eastern Gateway Building \\ Uxbridge, Middx, UB8 3PH, UK \\ Anatoli Djanatliev \\ Computer Networks \& Communication Systems \\ Computer Science 7 \\ University of Erlangen-Nuremberg \\ D-91058 Erlangen, GERMANY
}

\author{
Navonil Mustafee \\ The Business School \\ University of Exeter \\ Streatham Court, Rennes Drive \\ Exeter EX4 4ST, UK
}

\author{
Sally Brailsford \\ Southampton Business School \\ University of Southampton \\ Highfield \\ Southampton, SO17 1BJ, UK
}

\author{
Martin Kunc \\ Warwick Business School \\ Scarman Road Building \\ Scarman Road \\ Coventry, CV4 7AL, UK \\ Andres F Osorio \\ Department of Industrial Engineering \\ Universidad Icesi \\ Calle 18 No. 122-13 \\ Cali, COLOMBIA
}

\begin{abstract}
The last 10 years have witnessed a marked upsurge of attention on Hybrid Simulation (HS). The majority of authors define HS as a joint modelling approach which includes two or more simulation approaches (mainly Discrete Event Simulation, System Dynamics and Agent Based Simulation). Whilst some may argue that HS has been in existence for more than 5 decades, the recent rise tended to be more problem driven rather than technical experimentation. Winter Simulation Conference (WSC) 2015, 2016, 2017 have witnessed 3 panels on the purpose, history and definition of HS, respectively. This paper reports on a comprehensive review conducted by the panelists on HS and its applications. The aim of the paper is to move the debate forward by exploring potential platforms for developing concrete avenues for research on HS in conjunction with the modelling life cycle.
\end{abstract}

\section{INTRODUCTION}

The term Hybrid Simulation (HS) was in use since the early beginnings of Simulation as a discipline. The initial definitions were based on combining discrete variables models with continuous variables models, or even models that combine simulation with analytical methods such as optimization (Shanthikumar and Sargent, 1983). Most of the earlier attempts of HS were based on random and/or pragmatic interests from computer programming or analytical perspectives. In the last 10 years, however, there was a sharp rise in 
the interest on HS. This trend is confirmed by the review statistics shown in Figure 1. This is mainly driven by the rising need to cope with the heightened complexity of modern problems. This also gave rise to some confusion about the definitions and purposes of HS. To this end, WSC 2015, 2016, and 2017 have exhibited three panels on HS, discussing the purpose, history and definition of HS, respectively. This led to defining HS as the combined use of two or more simulation models from three main approaches (discrete event simulation (DES), system dynamics (SD), and agent based (ABS)). Given the ever rising complexity of the modern world, Hybrid Simulation and hybrid systems modelling approaches are both becoming important streams within the Modeling and Simulation arena. However, and despite the continuous effort, there remain three main challenges: first, there are no clear and cohesive definition for HS (Lynch et al. 2015); second, confusion between hybrid simulation and hybrid modelling study (Mustafee et al. 2015); third, there is a lack of a specific philosophical view point that defines HS as an independent approach (Shanthikumar and Sargent 1983). In this panel paper we aim to move the debate forward by reporting on a comprehensive review of the field of HS. The review was conducted by the panelists to come up with more refined definitions and uses of HS based on existing literature. The core of the review is focused around an agreed definition of the Simulation lifecycle and how HS approaches behave along the stages of the lifecycle. In this panel paper, each panelist leads the debate within each of the phases of the Simulation lifecycle which detailed below.

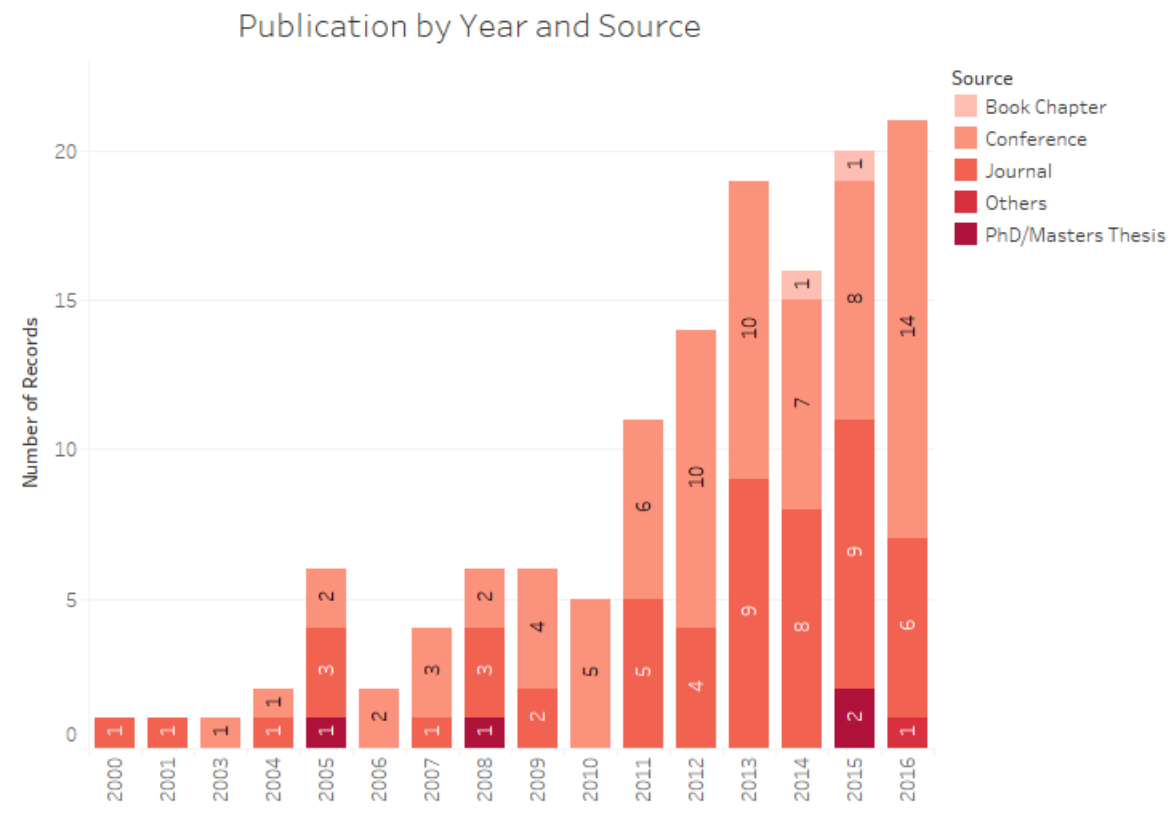

Figure 1: Evolution of HS literature over time, by source

\subsection{Simulation lifecycle}

There are a number of propositions of what a typical Simulation lifecycle is. For the purpose of the review and this panel, we adopt the lifecycle definition proposed by Brooks and Robinson (2000) as shown in Figure 2. This lifecycle is based five stages: Stage 1-Real World Problem Definition; Stage 2Conceptual Modelling; Stage 3-Computer Modelling; Stage 4-Verification and Validation; and Stage 5-Solution and Understanding. These stages will be detailed in the subsections below, however, the reader is reminded that, although this lifecycle assumes sequential stages, the process is not as linear when it comes to implementing it (as illustrated in Figure 2). 


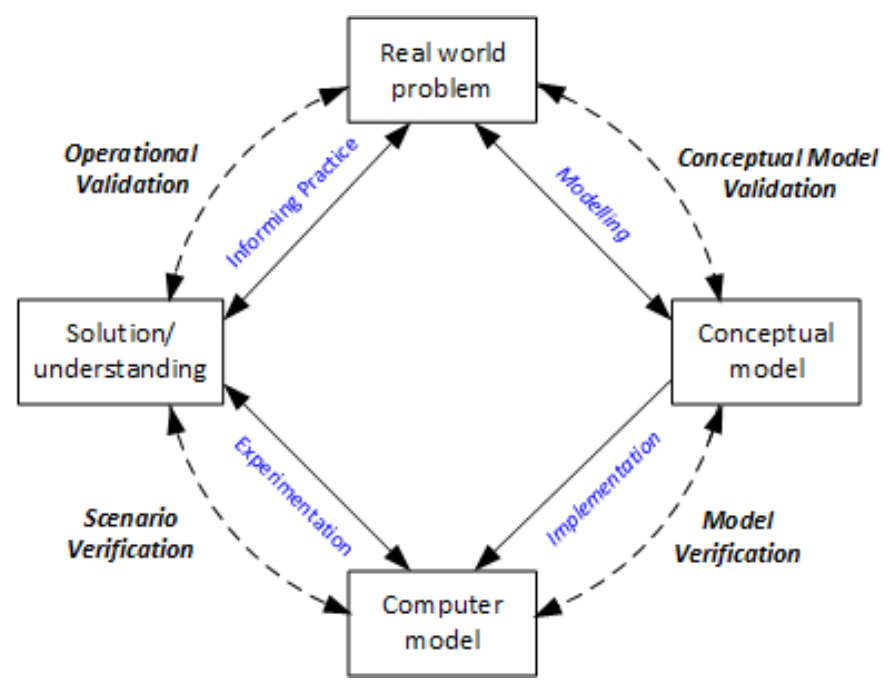

Figure 2: Stages of a simulation study, Adapted from Brooks and Robinson (2000).

\subsubsection{Stage 1: Real world problem}

The real-world problem usually determines the need for a specific solution and/or the need to improve understanding about it. This stage is divided into three main categories in the review:

- Application Area: denotes the general field of focus (e.g. healthcare or manufacturing systems).

- Application Context: denotes sub-application area (e.g. emergency department within healthcare).

- Type of hybrid study: has three types: Type A: models built for specific applications (case studies); Type B: case studies but also include generic frameworks; and Type C: purely theoretical, conceptual, or methodological.

\subsubsection{Stage 2: Conceptual Modelling}

A conceptual model is supposed to be an abstraction of the real world problem and is developed and validated with problem owners and/or domain experts. In a typical HS study, a conceptual model could be an integrated schema that describes the "objectives, inputs, outputs, content, assumptions and simplifications of the model" (Robinson, 2008) without reference to specific modelling techniques, or it could be composed of sub-models relevant to the approaches used (e.g., DES and SD) with simple links between them.

- Type of model: denotes the simulation approaches used (SD + DES, SD + ABS, DES + ABS, and $\mathrm{SD}+\mathrm{DES}+\mathrm{ABS})$.

- Type of hybridization: describes the relationship between the different submodels used and the data flow between them. Such data flow takes 4 possible forms - as stated in Morgan et al. (2017): enriching, sequential, interaction, and integration.

\subsubsection{Stage 3: Computer Modelling}

The purpose of the computer model is to translate the abstraction of the conceptual model in codes to enable modelers to experiment with different perspectives of the problem in a dynamical way. This stage is divided into the following categories in the review:

- Number of commercial packages used: how many commercial simulation packages (CSPs) used. 
- Names of commercial packages used: to show the most popular combination of CSPs for the development of specific hybrid model types.

- Programming languages: Most CSPs include support for a programming language. For example, Simul8 has Visual Logic and AnyLogic has support for Java.

- Model integration process: divided into three main possible integration process: Automated integration (contained within a CSP); Manual integration (copying and pasting data from one package into another); Integration using intermediate tools, such as Excel/VBA and HLA RTI.

- Model input source: divided into three categories: real world (primary or secondary), illustrative (experts opinions and guestimates, and mixed.

\subsubsection{Stage 4: Verification and Validation}

During verification, the computer model is assessed to ensure that it is a correct representation of the conceptual model. The process of validation ensures confidence in the model by both modelers and stakeholders and instilling trust in its outcomes. Model validation is rather a complex step, it comes in two possible ways: statistical approach and face validity.

\subsubsection{Stage 5: Solution and Implementation}

This aims to show that the simulation study has influenced real change using the intended solution. Intended solution could be a proof of concept, potential but not actual, real world implementation with clear implementation to solve the problem. The level of implementation is then measured according to the intended solution.

\section{STAGE 1: FINDINGS, CHALLENGES AND OPPORTUNITIES (ANDRES F OSORIO)}

One of the interesting aspects found is the specialization of countries in the different areas of application. While the USA presents the largest number of publications in Supply Chain Management, Transportation and Logistics and Manufacturing, the UK is the leader in publications in Healthcare and Canada in Construction and Civil Engineering. This information is presented in Table 1.

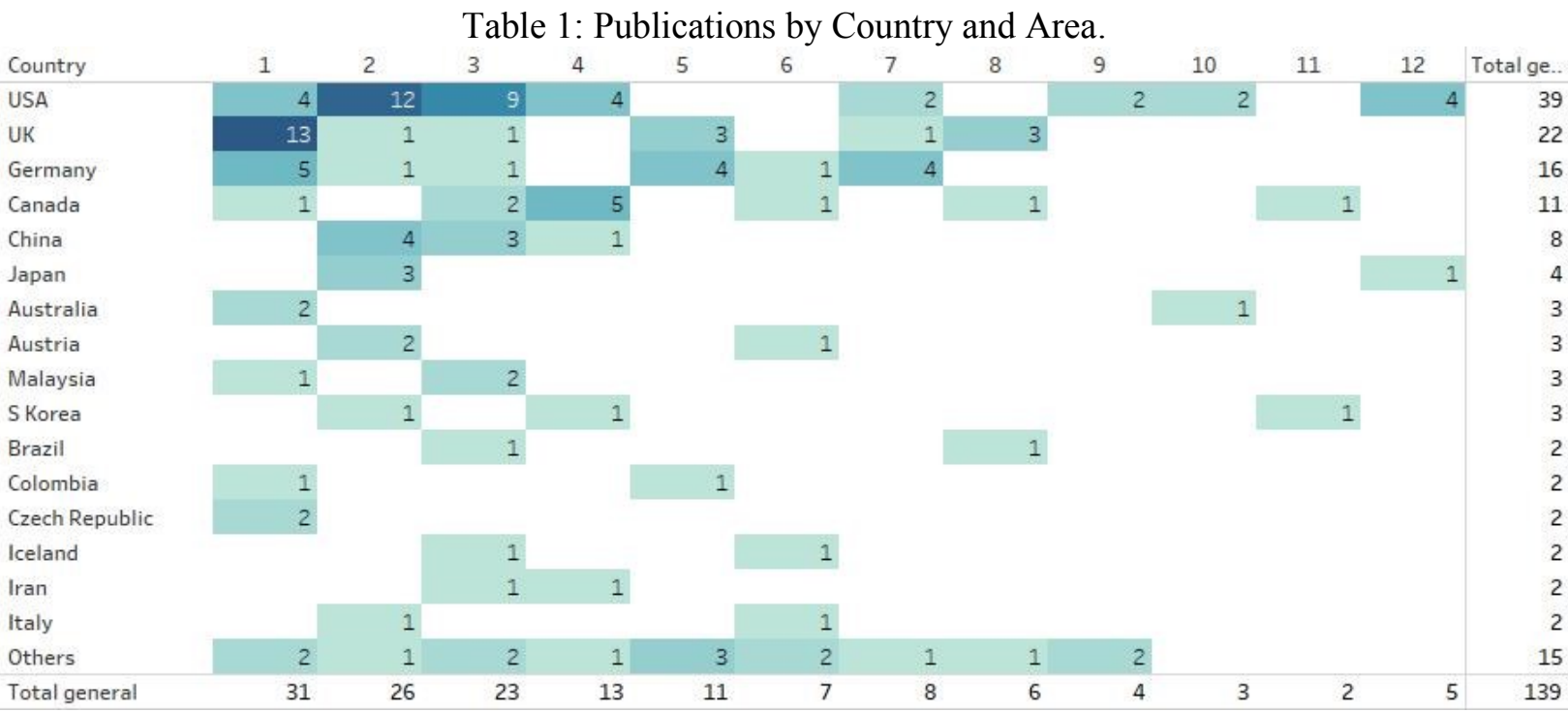

1: Healthcare, 2: Supply Chain, Transportation \& Logistics, 3: Manufacturing, 4:Construction \& Civil Engineering, 5: Business \& Management, 6: Ecology \& the Environment, 7: Energy, 8: Simulation, 9: Defense/Security, 10: Software development, 11: Project Management, 12: Other 
The range of applications is broad, however, a large portion of the publications found are concentrated in Healthcare, Supply Chain Management (including transportation and logistics), Manufacturing and Construction. The reason for this is perhaps the kind of problems found on these domains and the capacity of HS to represent complex problems. For example, in Healthcare it is usual to model ED systems using DES. However, this area is closely related with many other areas in a hospital such as diagnosis images, ICU and surgery. Given this, it is often required to simulate other areas probably in a different level of detail. Hence, other simulation paradigms can be useful. Furthermore, in supply chain management and manufacturing is usual to find DES models to represent the manufacturing stage but also models tend to use SD to represent other echelons of the supply chain or even other hierarchical levels in the same stage. In construction for example, DES is also used to represent the detailed operations of equipment and construction activities but other paradigms such as SD or ABS are used to represent external factors such as weather or individual behaviors of the operators. These are just examples of the complexities of these systems and an attempt to explain why HS is one of the most suitable methodologies to address problems on these domains.

\subsection{Type of Hybrid Study}

We classified the papers in three categories according to its content; Conceptual papers, papers that contain both a framework but also an application in a defined field and just applications. Since the previous is focused on the application fields, we present the more relevant findings for the two first categories.

\subsubsection{Conceptual/methodological/theoretical}

The integration of multiple simulation paradigms is challenging and several theoretical and philosophical aspects should be considered. Several papers have been published to discuss different these aspects. Morgan et al. (2011) develop a discussion of different aspects of using multiple methodologies. Two important aspects are reviewed: the relationship between methodologies and a sequence of steps to carry out a hybrid model. The relationship between multiple methodologies can be classified in isolationism, parallel, sequential, enrichment, interaction and integration while the steps mentioned are the following: problem and system exploration, select issue \& examine characteristics, designing combining and model development. Alzraiee et al. (2012) presents a framework to synchronize the execution time. This methodology is focused in three main aspects: The time advancing strategy, the DES advancing algorithm and finally the message sequence algorithm. This methodology is based on previous algorithms to control parallel and distributed simulation. Jacob et al. (2010) introduce again the concept of hybrid simulation, however, uses a different language and calls micro and macro systems. The paper starts the general explanation for systems with continuous and discrete behaviors and then proposes mechanisms to handle the time transitions between SD and DES as well as the method to couple the model components. Brailsford et al. (2010) discuss the advances of hybrid modelling using two examples of applications of HS in healthcare. Some of this theoretical aspects are closely related with the solution software used. For instance time transition times can be often automatically handled by Anylogic. This is similar to the control pf the sequence of execution for the different models. However, in other solution packages or in the integration of two specialized simulation software (for instance DES and SD) the solution approach must be considered carefully.

\subsubsection{Application and Framework}

Several frameworks have been proposed in the literature to help modelers in the development of hybrid models in specific fields. In this section we describe some papers that have been developed within this scope. In Chahal and Eldabi (2008) a framework composed by three stages is proposed to determine the necessity of hybrid simulation, the relationship of the interaction points and the mode of interaction. 
Necessity of hybrid simulation is analyzed using the objectives of the simulation and questioning whether this can be achieve using just one paradigm or independent models. Alvanchi et al. (2011) also presents a methodological framework to combine SD and DES. Firstly, the authors state that the main challenges in hybrid simulation are the lack of modelling frameworks, the time advancing strategy and the communication architecture. Helal and Rabelo (2006) presents a paper aimed at studying hybrid modelling in manufacturing. This paper is mainly focused in the development of a controller to link strategic levels in decision making using an SD model and tactical and operational levels represented by a DES model. Arena and Vensim have been employed for each simulation paradigm. Finally, Shi-zhen \& Wen-li (2007) present a paper aimed at studying the impact of transportation disruption in supply chains. In this paper the authors compare a traditional supply chain and a VMI supply chain. The supply chain modelled considers different sectors such as sectors: retailer, distributor, manufacturer and manufacturer's production department. Production is modelled as continuous while transportation and ordering are considered discrete elements. As presented, several attempts to clarify theoretical aspects of HS have been developed, however, despite these publications there is not a unified framework to develop HS regardless the application field.

\section{STAGE 2: FINDINGS, CHALLENGES AND OPPORTUNITIES (MARTIN KUNC)}

Conceptual modelling is a vitally important step in the modelling process (Robinson, 2008), and overall more than $60 \%$ of the papers contained some evidence that a conceptual model had been developed. However, the practice of conceptual modeling is not following any specific standards. Some papers follow a discussion of the problem situation and the corresponding objectives, inputs, outputs and simplifications of the whole hybrid model like a discrete event model, e.g. (Wallentin and Neuwirth, 2017). In other papers, a discussion of the modules comprising the hybrid model is their way of presenting the conceptual model, e.g. Shafiei et al. (2013). In a set of papers we found models using some specific graphical representation (e.g. causal loop diagrams, state flows, activity diagrams) is their way of representing the conceptual model, e.g. Jo et al. (2015). Even using these very simple definitions of conceptual modelling, conceptual modelling for hybrid models as a whole is not well-developed. Individual simulation methods have their own bespoke graphical approaches to represent conceptual models, such as causal loop diagrams for SD, statecharts for ABS and process flow or activity diagrams for DES, but while these are well suited for that particular method they do not have obvious capabilities to model the hybridization elements (Brailsford et al. 2018). While conceptual models for individual models belonging to a hybrid model is important, these were presented independently and did not provide a clear explanation of factors such as information exchange or hierarchy in terms of model dominance.

Overall, we did not find any specific conceptual modelling approaches to support hybrid simulation, and this is an area where strong work is needed to make HS a key component in the simulation community. Then, what hybrid modelers have to do? All three individual simulation methods have a recognized set of steps for model development, starting with problem definition and ending with informing practice, as described for example by Sargent (2007) and Brooks and Robinson (2000). While this "project life-cycle" was designed for DES, it can be applied equally to SD and ABS. Although the emphasis on different stages may vary, developing a hybrid model does not necessarily deviate greatly from this process. In fact, individual submodules (or models) often closely follow their own established processes.

Nevertheless, the development of a hybrid model adds a vital extra component to this process: the links between submodels. How the different submodels are expected to work together? What information should be exchanging and when? Are they synchronous or asynchronous? However, there is an important issue: how to link a process flow model, a state chart, and a stock flow model. Moreover, a further technical issue is how computer models should be linked at runtime. Even in a multi-method modelling environment like AnyLogic, the flow of information between components has to be considered. Therefore, conceptual modeling is key and it should be specified at the beginning of any modeling effort.

We believe that a generalized framework for model development in hybrid modelling should not focus on the individual models, but rather on the links between them. This will involve information about how 
the components to be linked, and the information to be exchanged. This is a promising area for future research. Some aspects to consider in this future research are:

- How to capture the problem situation in terms of the subsystems that comprise it,

- How to provide a rigorous and systematic way to identify the characteristics of each subsystem that indicate the use of a given simulation method

- While, the choice of method is currently usually made by the modeler based on their disciplinary background or preference, and their expertise, a key feature of a HS methodology must be an ability to evaluate the benefit of each method (SD, DES and ABS) for each subsystem and then finally, on a parsimonious principle, determine whether one method would suffice for all (and if so, which). Therefore, the key question in the model conceptualization stage is: why a hybrid model is needed for this problem situation?

- The methodology should facilitate a description of the links and data exchange mechanisms between subsystems, ideally in graphical form, and should also provide the modeler with some kind of checklist of all the software-independent issues that need to be considered when linking a model using a certain method with a model using another method

\section{STAGE 3: FINDINGS, CHALLENGES AND OPPORTUNITIES (NAVONIL MUSTAFEE)}

In the context of a hybrid simulation study, this stage consists of model implementation using either one or more commercial simulation packages or through the use of programming languages and existing modelling libraries.

\subsection{Commercial Simulation Packages (CSPs)}

Our review has identified three main CSPs that support more than one simulation technique - AnyLogic, ExtendSim and NetLogo. The majority of the papers reviewed have used AnyLogic, and it has been applied for all four hybrid modelling variations, namely, SD+DES, SD+ABS, ABS+DES and SA+ABS+DES. The predominance of AnyLogic is not surprising since having a common simulation executive for ABS, DES and SD has its advantages, e.g., common representation of time and inbuilt mechanisms for data-exchange. Arena and Simul8 also appear in the list of top five CSPs. Both packages have largely been used with SD CSPs like Vensim and iThink. The third most popular CSP on our list is Vensim, and it has only been used with DES CSPs like Flexim, Arena and Simul8 to develop hybrid SD+DES models. We found ExtendSim and NetLogo to be the fourth and fifth most popular CSPs.

\subsection{Programming Languages}

These findings relate to studies that have specifically referred to model implementation but have had not mentioned a CSP. We found 11 studies that referred to implementation in programming languages and modelling libraries. From the departmental affiliation of the first author of these publications, we note that most of the departments are associated with either Engineering (e.g., Industrial Engineering, Civil/Construction Engineering) or Computer Science. This is perhaps not surprising since programming tends to be among the core subjects in such disciplines. Bergman et al. (2008) have used MASON library to develop a hybrid ABS-SD model for capturing transitions in socio-technical systems towards more sustainable development. MASON is a discrete-event multi-agent simulation library for developing simulations in Java https://cs.gmu.edu/ eclab/projects/mason/. Their model includes complex agents, which have an internal system dynamics model. Srivastava et al. (2005) propose a modular simulation environment, developed in Pythagoras ABM (Henscheid et al., 2006), to assess the impact of new technology in manning costs for naval vessels. 


\subsection{Model Integration Process}

One critical aspect of hybrid simulation is the integration process between the different tools. We have considered three main strategies for the integration process: manual integration (copying and pasting data from one package into another), integration using intermediary tools (e.g., using Excel/VBA and HLA RTI) and automated integration (enabled through a CSP which supports multiple simulation techniques). Automated integration has been reported in 67 studies, of which 47 studies have used AnyLogic, six studies refer to ExtendSim and four studies include NetLogo implementation. The remaining studies mostly comprise of bespoke solutions using programming languages and simulation libraries. 23 papers have reported on using an integration strategy that relies on intermediate tools like distributed simulation software (e.g., HLA-RTI and PoRTIco RTI with corresponding $\mathrm{C}++/$ Java bindings), programming languages (e.g., Excel/VBA and VB .NET) and databases for information exchange (e.g., MySql server). Although manual integration has been reported only in five studies, it is arguable that papers that have implemented a model but have not specifically reported on an integration strategy (and thus classified as 'not reported'), would have included some form of manual integration. Furthermore, some papers that have been presently been classified as integration using intermediate tools, could also include an element of manual integration. Overall, only a few authors have articulated the integration process for models developed using different CSPs.

\subsection{Model Input Source}

We identified three categories of input data:

- Real world data source: Data from case study (including primary data collection and retrieval of data from information systems) or secondary data. Some input data may also be estimates.

- Illustrative data source: These are estimates from domain experts or data for illustrative purposes only with no justification.

- Mixed data source: This is the combination of the first two categories, i.e., real world data and illustrative data.

For this analysis, we considered 100 papers which discussed the input data for their model (see Figure 3). Of these, 40 used purely illustrative data. Approx. 20 of these 40 papers described applications where ABS was one of the methods used. Arguably, this explains the use of illustrative data since the collection of empirical data for modelling the decision rules governing agent behavior is not a simple matter. It is also unsurprising to see papers that present frameworks using illustrative data, since a paper that presents a framework is less likely to be focused on a real-world problem. Around 20 papers contained a combination of both real world data and illustrative data ("Mixed Data Source" in Figure 3). The reason for using mixed data is related with the use of HS to model systems at multiple levels. For example, a DES model of a factory production line may use real-world data routinely collected from operational reporting systems, such as machine processing times and breakdown frequencies, but the data required to model more strategic aspects such as long-term feedback effects or policy making (the natural realm of SD) are more difficult to obtain. Only 39 papers were based entirely on real world data, which provides further confirmation of the lack of real-world applications of HS. The category "Other" relates to one paper in which it was impossible to determine the nature of the data source. 


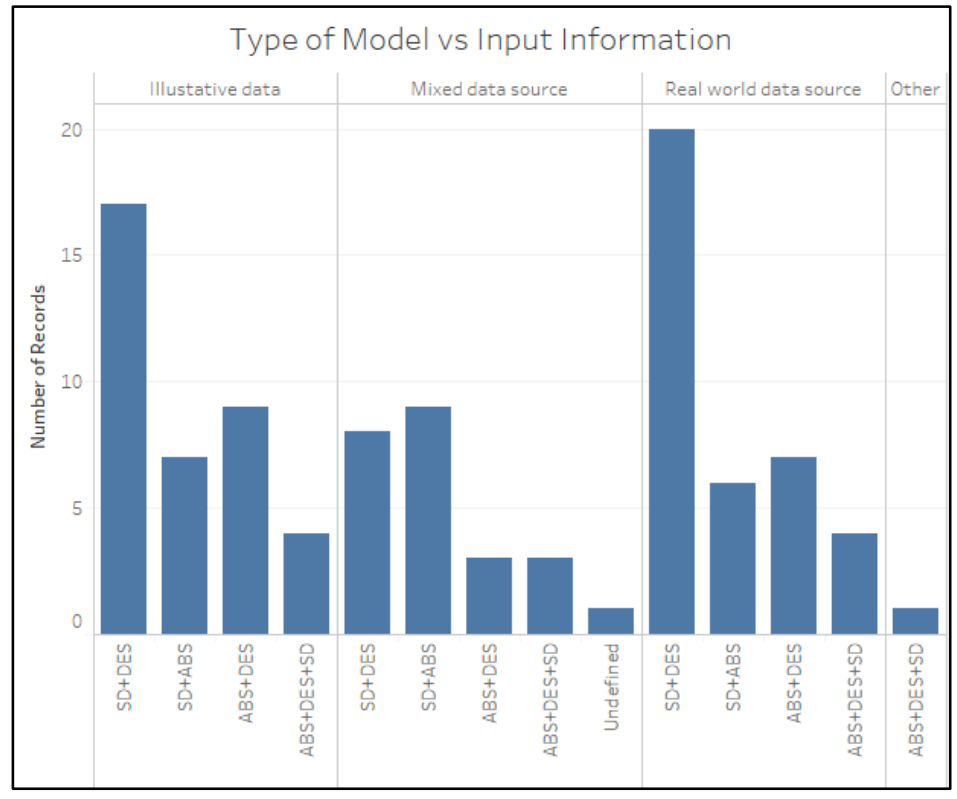

Figure 3: Type of model and the associated input data source.

\section{STAGE 4: FINDINGS, CHALLENGES AND OPPORTUNITIES (TILLAL ELDABI)}

Although it may be disappointing, it was not entirely surprising to see that most of the reviewed papers in HS did not explicitly report any kind of verification or validation. Of those papers that claim to have modeled real world problems, only a small minority presented descriptions of both verification and validation, in fact only one paper used standard statistical methods. In the models where there were evidence of verification and validation, it was noticeable that most of the verification and validation approaches found were "intra-modular", i.e. each individual submodel was evaluated separately in accordance with the approaches used for that particular model. There were no evidence of verification of links between submodels, i.e. "inter-modular" verification and validation. Only a handful of authors have attempted to provide more comprehensive propositions for verification of HS models, that is inter-modular verification. For example, Hwang et al. (2016) introduced an intermediate model between the SD and DES models to assess the credibility of the exchanged information by matching it with the expected values. Ali et al. (2014) follow a similar approach for inter-modular verification for HS between ABS and SD.

A major lesson learned from the review is that the process of intra-modular verification and validation submodels is similar to the existing approaches for single-method models. However, inter-modular validation proved more complex. That is, if formal validation of standalone SD or ABS models is difficult enough, it becomes infinitely more so when the a SD or ABS model is combined as a hybrid with another kind of simulation method.

\subsection{Challenges to Validation of HS Models}

Perhaps the most obvious challenge associated with validating HS models is the diversity of approaches needed. That is to say, validation of quantitative numerical models, e.g. DES, requires statistical methods. Whilst, validation of qualitative models using verbal or causal theories, e.g. SD and ABS, implies validation during the modelling process. Validation of SD models typically involves establishing face validity and involving stakeholders in all stages of the model development process, as well as aspects such as checking dimensional consistency or performing extreme value tests. ABS models are often based on assumptions and beliefs about the micro-level relationships between model elements and psychological behavioral rules, 
which may produce aggregated results that mirror observed data but can never be validated statistically at the individual level. There is an added challenge for HS when it comes to inter-modular verification of the exchanged information. This is an area where further research aimed at developing more rigorous methods is urgently needed. Another challenge, highlighted in Ali et al. (2014), is that modelers need to be familiar with the relevant verification approaches for each method. For example, it is not possible to verify SD policy models or ABS models of emergent behaviors using methods developed for quantitative, predictive DES models. It is somewhat normal to find that only few studies use statistical methods for validation, however, it was surprising to see that only how few have used face validity. This is clearly an area where further research is urgently needed.

\subsection{Research Opportunities in Verification and validation of HS Models}

While statistical validation of ABS and SD models may not be widespread, it is increasingly becoming as important (Rahmandad et al. 2015). New methods for validation must be developed if real-world decisionmakers are going to have confidence in the results of HS models. The question of client trust, which is difficult enough in a DES model with an attractive animated visual display, is even more challenging in a hybrid model "glued together" with computer code. Meanwhile, modelers should do all they can do address these issues by applying standard methods to the verification and validation of the individual component submodels, and at least perform (and report) some kind of basic face validity sense-checking or extreme value testing to the whole hybrid model.

\section{STAGE 5: FINDINGS, CHALLENGES AND OPPORTUNITIES (SALLY BRAILSFORD)}

In the UK, measuring "impact" now forms a substantial part of the Government's Research Excellence Framework (REF), an exercise conducted every five or six years to determine which universities should be funded to do research and by how much. In this context, impact does not mean academic impact or citations, but real-world impact: demonstrable change taking place in an organization or, depending on the nature and scope of the research, the wider environment. In the REF, impact is not measured merely in financial terms but can take many forms: societal, political, health and wellbeing, environmental, and cultural.

Despite the increasing importance of impact in assessing the quality of research, the sad fact remains that even in a supposedly problem-driven discipline like simulation and modeling, only a small fraction of published papers actually report that the model described therein has had a tangible impact in the real world or has been used to inform a real-world decision. For example, Katsaliaki and Mustafee (2011) both found that just over $5 \%$ of published papers in the field of healthcare-related simulation modeling actually reported that the model findings had been used in practice. In the case of hybrid simulation, the situation is even worse: it is almost impossible to find a paper describing a real-world implementation.

Brailsford et al. (2009) suggest that one reason for the lack of reported model use is the fact that the "publish or perish" nature of academic life forces researchers to publish their work before there has been time for it to be implemented in practice. Even if a model is developed in collaboration with a hospital or some other healthcare organization in order to address a specific problem, it can often be a very long time before the real-world change takes place and the effects measured. Another reason is that simple solutions based on simple models are far likely to be implemented, but it is very hard to publish standard applications of discrete-event simulation (DES) or system dynamics (SD), especially in the top journals. Methodological innovation is required, and new methods are far less likely to be trusted by "clients" or collaborators than simple models using tried-and-tested methods like DES or SD.

In the case of hybrid simulation, this difficulty is amplified. Most models are developed by academic researchers, sometimes for genuine practical reasons but often just out of curiosity. The literature (including many papers presented in the HS track at WSC since its inception in 2011) shows that the overwhelming majority of HS models that allegedly model a real problem situation are actually used by academics to experiment with potential solution strategies, rather than by practitioners and managers as decision support. While this partly reflects the fact that HS is a comparatively new field, and is still in the very early stages 
of establishing itself as a valid modeling approach, more research needs to be done to identify the reasons for this low level of implementation. One potential reason is the lack of suitable data at the correct level of aggregation (and disaggregation); another is the lack of a formal process for developing a conceptual model; a third possible reason is the lack of established methods for documenting the hybridization process. Many published papers do not even contain a diagrammatic representation of the linkages between submodels, and this is clearly a barrier to understanding by lay users. Compare this situation with the impressive visual graphics in most commercial DES software tools.

Clearly, many HS models have the potential for enormous impact and have strong practical relevance. The reason for the near-exponential increase in the popularity of HS in the 21 st century is that most realworld problems and systems are complex, with many different aspects, levels and perspectives, and it is rare that one single method is ideally suited to capture all of them. The modeler who is only familiar with one method is therefore faced with a dilemma. Is it better to model the whole problem/system using their favorite method, accepting that it oversimplifies some aspects (or worse, makes invalid assumptions for the sake of mathematical tractability), or to model only those parts of the problem for which their chosen method is suitable and simply say that the remaining parts are out of scope? The former approach may lead to poor solutions (and bad decisions), but from a practical perspective it may be neither useful nor sensible to study only one aspect of a real-world problem in isolation.

Software maturity is highly relevant. Compared with DES, and even with SD, there are relatively few agent-based software packages. Most of the available tools (e.g. Netlogo and Repast) were primarily developed for academic research purposes and hence building models in them involves writing a lot of code. While this obviously provides great flexibility (and also means they can be more easily combined with aspects of DES or SD), these packages are rarely taught outside computer science degree programs. The first, and still the only, commercial software tool that was purposely designed from the start to allow modelers to develop practical hybrid simulation models in all three paradigms is AnyLogic (www.anylogic.com) and while this now has a nice graphical interface that allows the user to drag-anddrop icons on the screen and use dialog boxes to enter model parameters, etc, developing anything more than a fairly simple model still requires some facility in writing Java code.

Of course, the academic literature is only one measure of the practical use of any modeling approach. As yet, hybrid simulation is not routinely used by practitioners: AnyLogic is gradually changing this, as anyone who visits their website and looks at their list of clients can see, but it will not happen overnight. Maybe in ten years' time, when a new generation of students have left university having been taught simulation using only AnyLogic, and will ask their employers to buy it, things might be different. However today, the decision to develop a hybrid model should not be taken lightly, especially in a consultancy setting where there is a client who needs a quick answer.

\section{LESSONS FROM REAL LIFE HYBRID SIMULATION MODELS (ANATOLI DJANATLIEV)}

Usually, many real-world problems can be modeled by one most appropriate simulation paradigm following the just described stages of the simulation lifecycle. Flow-based structures can be best represented by SD, while state-charts are well-suited to model discrete decisions/actions applying DES techniques. Agentbased models (ABM) have the power to represent individual actions of agents emerging a global systemlevel behavior. Even complex and large-scale problems can be modeled by only one dedicated simulation technique, however, this process can be very challenging, or in words of Brailsford et al. (2010), it can be compared to "a case hammering in a screw." This section focuses on selected HS lessons from case studies in healthcare and mobility with real stakeholder involvement. In healthcare we particularly developed largescale hybrid models to answer "what-if?" and "how to?" questions within the context of prospective health technology assessment (Djanatliev and German 2013). 


\subsection{Large-Scale Modeling - Challenges in the Life-Cycle}

Large-scale simulations are not only faced with the problem of paradigm selection. Following the lifecycle, the first challenge is often to find a common "language" for all participating stakeholders (e.g., domain experts, data experts, technical experts) in order to represent the problem and to define common aims for a case study. Domain experts (e.g., physicians in healthcare) are usually focused on the problem to be solved and not on the modeling paradigm which is the main task of simulation experts. The conceptual modeling is therefore an important step before implementing a running model and selecting an appropriate simulation technique. At this stage, we learned that it can be very helpful to draw the situation by an abstract representation, such as a non-formal graph consisting of simple nodes and connections (e.g., in MS Visio). This approach similarly encourages all stakeholders to proceed problem-oriented instead of developing a model based on a previously selected simulation method. We call this abstract graph representation the Conceptual Domain Model (CDM).

Another important aspect in large-scale modeling is the partitioning of a complex problem in smaller units (sub-problems). This modularization procedure helps domain experts to focus only on those parts of the problem that are best-represented by their knowledge. For example, a physician can be involved in healthcare specific sub-modules while an economic expert mostly focus on health-economic aspects of the overall problem. Once modules have been identified, their integration in a global context must be done. Until this stage we had no necessity to distinguish between simulation paradigms. Going one step further towards the implementation stage, the previously identified modules can be modeled by appropriate simulation techniques. Using a CDM as a basis, a more formal representation can be derived, the Formal Conceptual Model (FCM), which includes all identified modules and already considers the selected simulation paradigm. This representation itself serves as a basis for the model implementation stage. However, how we can represent problems that are on the one hand flow-based and on the other hand triggered by discrete decisions? The solution therefore is Hybrid Simulation. A representative example for this situation are energy flows that can be affected by discrete actions, e.g., switching lights on/off.

\subsection{Best Practices and Opportunities}

Hybrid Simulation is not just a tool to combine continuous and discrete structures. It gives rather the opportunity to develop models problem-oriented and more flexibly. Thus, large-scale models are able to run faster by using hybrid techniques. In this case, we usually start modeling by abstract SD models having low data requirements and we "zoom-in" more important model parts. Following this top-down approach, we use only data that we truly need. Different levels of abstraction help to solve trade-off decisions between model accuracy and simulation performance. In Djanatliev and German (2015), we have presented best cases that can be applied in hybrid case studies. In particular, we described five types of interaction between continuous and discrete models. For example, the combination between SD and ABM consist mainly of the generation of agents from continuous models. Otherwise, the behavior of agents can be modeled by SD. An example therefore are biological body processes of humans (represented by agents). Taking medication can affect those internal processes. Finally, in order to develop more performant models, single agents with similar parameters can be combined in common "superagents".

All these methodological aspects can be realized, if appropriate software packages exist. As already mentioned, one option is to combine single paradigm modeling tools. Another possibility is to use powerful multi-method simulation packages that allow to combine different simulation techniques in common environments. AnyLogic is the most important example that we used successfully to implement hybrid models together with all involved stakeholders.

\section{SUMMARY}

This paper presented views from six panelists on the current status of HS approaches and uses in conjunction with the Simulation lifecycle, which is based on the following stages. Stage 1-Real World 
Problem Definition; Stage 2-Conceptual Modelling; Stage 3-Computer Modelling; Stage 4Verification and Validation; and Stage 5-Solution and Understanding. The views of the panelists are focused on extracting findings, challenges, and future research prospects for HS for each of the aforementioned five stages. These views are mainly generated from a comprehensive literature review of HS approaches and uses, which is conducted by the panelists with reference to the lifecycle stages. Based on our review, we propose HS should follow Figure 2 process as a top-level framework to conduct, organize and report HS studies. Then, each stage can contain diverse methods and frameworks depending on the characteristics of the study. Therefore, we propose a set of observations and recommendations linked to each stage in the lifecycle as shown in Table 2.

Table 2

\begin{tabular}{|c|c|}
\hline Stage & Observations/Recommendations \\
\hline $\begin{array}{l}\text { Real World } \\
\text { Problem }\end{array}$ & $\begin{array}{l}\text { - There direct relations between problem complexity and need for HS } \\
\text { - HS is used more in applications areas where simulation methods have } \\
\text { extensive use }\end{array}$ \\
\hline Conceptual Model & $\begin{array}{l}\text { - Overarching system perspective based on the abstraction of the real } \\
\text { world problem } \\
\text { - Modules for the Hybrid model and rationale for the use of SD, ABS or } \\
\text { DES } \\
\text { - Type of hybridization and linkages between models }\end{array}$ \\
\hline Computer Model & $\begin{array}{l}\text { - HS tools selection is dictated by ease of linkages } \\
\text { - Single all-encompassing packages tend to be used more for HS } \\
\text { - Non-uniformity of data sources is a significant challenges for HS model }\end{array}$ \\
\hline $\begin{array}{l}\text { Validation and } \\
\text { Verification }\end{array}$ & $\begin{array}{l}\text { - Intra-modular VV methods are similar to single models } \\
\text { - Inter-modular VV methods add extra dimensions to validation } \\
\text { - } \quad \text { Non-uniformity of testing methods require more effort and skills }\end{array}$ \\
\hline $\begin{array}{l}\text { Solution and } \\
\text { Understanding }\end{array}$ & $\begin{array}{l}\text { - Simulation packages are below threshold to meet demand complexity } \\
\text { - Trade-off between skills-mix and method selection is critical } \\
\text { - HS is still an academic pet subject and needs to be more industrially } \\
\text { accessible }\end{array}$ \\
\hline
\end{tabular}

\section{REFERENCES}

Morgan, J., S. Howick, and V. Belton. 2011. "Designs for the Complementary Use of System Dynamics and Discrete-Event Simulation". In Proceedings of the 2011 Winter Simulation Conference, edited by S. Jain et al. 2710-2722. Piscataway, New Jersey: IEEE.

Alzraiee, H., T. Zayed, and O. Moselhi. 2012. "Methodology for Synchronizing Discrete Event Simulation and System Dynamics Models". In Proceedings of the 2012 Winter Simulation Conference, Edited by C. Laroquedoi et al. 3225-3236. Piscataway, New Jersey: IEEE.

Jacob, M., C. Suchan, and O. K. Ferstl. 2010. "Modelling of Business Systems using Hybrid Simulation A New Approach". 18th European Conference on Information Systems, ECIS 2010. Available at: http://aisel.aisnet.org/ecis2010/6.

Brailsford, S. C., S. M. Desai, and J. Viana. 2010. "Towards the Holy Grail: Combining System Dynamics and Discrete-Event Simulation in Healthcare". In Proceedings of the 2010 Winter Simulation Conference, Edited by B. Johansson et al. 2293-2303. Piscataway, New Jersey: IEEE.

Alvanchi, A., S. Lee, and S. AbouRizk. 2011. "Modeling Framework and Architecture of Hybrid System Dynamics and Discrete Event Simulation for Construction". Computer-Aided Civil and Infrastructure Engineering 26(2):77-91. 
Djanatliev, A., and R. German. 2013. "Prospective Healthcare Decision-Making by Combined System Dynamics, Discrete-Event and Agent-Based Simulation". In Proceedings of the 2013 Winter Simulation Conference, Edited by R. Pasupathy et al. 270-281. Piscataway, New Jersey: IEEE.

Djanatliev, A., and R. German. 2015. "Towards a Guide to Domain-Specific Hybrid Simulation". In Proceedings of the 2015 Winter Simulation Conference, Edited by L. Yilmaz et al. 1609-1620 Piscataway, New Jersey: IEEE.

Helal, M. and L. Rabelo. 2006. "Interactions of the Three Management Levels in the Manufacturing Enterprise System Using Hybrid Simulation". In IIE Annual Research Conference.

Shi-zhen, B. and W. Wen-li. 2007. "The Impact of Transportation Disruption on Adaptive Supply Chain: A Hybrid Simulation Study". Proceedings of 2007 International Conference on Management Science and Engineering 844-849.

Brooks, R. J. and S. Robinson. 2000. Simulation, Palgrave Macmillan.

Jo, H., H. Lee, Y. Suh, J. Kim, and Y. Park. 2015. A Dynamic Feasibility Analysis of Public Investment Projects: an Integrated Approach using System Dynamics and Agent-Based Modeling. International Journal of Project Management 33(8):1863-1876.

Robinson, S. 2008. "Conceptual Modelling for Simulation Part I: Definition and Requirements". Journal of the Operational Research Society 59(3):278-290.

Sargent, R. G. 2007. "Verification and Validation of Simulation Models". In Proceedings of the 2007 Winter Simulation Conference. Edited by S. Jain et al. 124-137. Piscataway, New Jersey: IEEE.

Shafiei, E., H. Stefansson, E. I. Asgeirsson, B. Davidsdottir, and M. Raberto. 2013. Integrated Agent-Based and System Dynamics Modelling for Simulation of Sustainable Mobility. Transport Reviews 33(1): 44-70.

Wallentin, G.and C. Neuwirth. 2017. "Dynamic Hybrid Modelling: Switching Between AB and SD Designs of A Predator-Prey Model". Ecological Modelling 345(2):165-175.

Hwang, S., M. Park, H. Lee, and S. Lee. 2016. "Hybrid Simulation Framework for Immediate Facility Restoration Planning after A Catastrophic Disaster". Journal of Construction Engineering and Management 142(8):1-15.

Ali, M., A. Dulcy, and D. Daniel. 2014. "Ex-Ante Policy Analysis in Civil Infrastructure Systems". Journal of Computing in Civil Engineering 28(5):1-14 .

Shanthikumar, J. G., and R. G. Sargent. 1983. "A Unifying View of Hybrid Simulation/Analytic Models and Modeling". Operations Research 31(6):1030-1052.

Lynch, C. J., and S. Y. Diallo. 2015. "A Taxonomy for Classifying Terminologies that Describe Simulations with Multiple Models". In Proceedings of the 2015 Winter Simulation Conference, edited by L. Yilmaz et al. 1621-1632. Piscataway, New Jersey: IEEE.

Mustafee, N., J. H. Powell, S. C. Brailsford, S. Diallo, J. Padilla and A. Tolk. 2015. "Hybrid Simulation Studies and Hybrid Simulation Systems: Definitions, Challenges, and Benefits". In Proceedings of the 2015 Winter Simulation Conference, Edited by L. Yilmaz et al. 1678-1692. Piscataway, New Jersey IEEE.

Henscheid, Z., D. Middleton, and E. Bitinas. 2006. "Pythagoras: An Agent-Based Simulation Environment". Available at: https://calhoun.nps.edu/handle/10945/35599 [Accessed May 23, 2018].

Srivastava, N., F. Pietryka, G. Horne, and M. Theroff. 2005. "Simulation Environment to Assess Technology Insertion Impact and Optimized Manning". In Proceedings of the 2005 Winter Simulation Conference, Edited by M. E. Kuhl et al. 1088-1093 Piscataway, New Jersey IEEE.

Bergman, N., A. Haxeltine, L. Whitmarsh, J. Köhler, M. Schilperoord, and J. Rotmans. 2008. "Modelling Socio-Technical Transition Patterns and Pathways". Journal of Artificial Societies and Social Simulation 11(3):7.

Morgan, J.S., S. Howick, and V. Belton. 2017. "A Toolkit of Designs for Mixing Discrete Event Simulation And System Dynamics”. European Journal of Operational Research 257(3):907-918. 
Chahal, K. and T. Eldabi. 2008. "Applicability of Hybrid Simulation to Different Modes of Governance in UK Healthcare". In Proceedings of the 2008 Winter Simulation Conference. Edited by S. J. Mason 1469-1477.

Rahmandad, A., R. Oliva, and N. Osgood. 2015. Analytical Methods for Dynamic Modelers, The MIT Press, Boston: USA.

Katsaliaki, K. and N Mustafee. 2011. "Applications of Simulation within the Healthcare Context". Journal of the Operational Research Society, 62(8):1431-1451.

Brailsford, S., P. Harper, H. Patel, M. Pitt. 2009. "An Analysis of the Academic Literature on Simulation And Modelling In Health Care”. Journal of Simulation 3(3):130-140.

\section{BIOGRAPHIES}

TILLAL ELDABI is senior lecturer at Brunel University. He has B.Sc.in Econometrics and M.Sc. and $\mathrm{Ph} . \mathrm{D}$. in Simulation Modeling in Healthcare. His research is into aspects of healthcare modeling and simulation. He developed a models support health economists and clinicians to decide on best treatment programs. He has published widely in the field of modeling in healthcare and edited a number of special issues in related journals. His email address is tillal.eldabi@brunel.ac.uk.

SALLY BRAILSFORD is Professor of Management Science at the University of Southampton, UK. She received a BSc in Mathematics from the University of London, and MSc and PhD in Operational Research from the University of Southampton. Her research interests include simulation modeling methodologies and the modeling of human behavior in healthcare systems. Her email address is s.c.brailsford@soton.ac.uk.

ANATOLI DJANATLIEV is an Assistant Professor (AkadR) at the Friedrich-Alexander-University Erlangen-Nuremberg. His research interests include various topics on combined simulation techniques in application areas healthcare and mobility. His e-mail address is anatoli.djanatliev@fau.de.

MARTIN H KUNC is an Associate Professor of Management Science at Warwick Business School, University of Warwick. He holds a PhD in Decision Sciences from London Business School, UK. His research interests lie in system dynamics simulation modeling, especially in healthcare, strategic decision making processes and dynamics of competitive industries. His email address is martin.kunc@wbs.ac.uk

NAVONIL MUSTAFEE is Associate Professor of Operations Management and Analytics at University of Exeter Business School. His research interests are in simulation methodologies, hybrid simulation, hybrid systems modelling and healthcare simulation. His e-mail address is n.mustafee@exeter.ac.uk and his webpage is http://sites.google.com/site/navonilmustafee/.

ANDRES F. OSORIO is lecturer in the Industrial Engineering department at the Universidad Icesi in Cali, Colombia, He received a BSc and MSc in Industrial Engineering from Universidad del Valle and $\mathrm{PhD}$ in Management Sciences form University of Southampton. His research interests include optimization and simulation applied to health systems and supply chain management. His email address is afosorio@icesi.edu.co. 